\title{
Views of Cesarean Birth Among Primiparous Women of Mexican Origin in Los Angeles
}

\author{
Laura H. Cummins, M.A., Susan C.M. Scrimshaw, Ph.D., and Patricia L. Engle, Ph.D.
}

\begin{abstract}
This study examined data from a larger project on the cultural context of first birth among low-income women of Mexican origin giving birth in Los Angeles. Data on knowledge of cesarean birth and perceptions of the cesarean birth experience were collected. In addition, differences in perceptions of the experience between women giving birth vaginally and those giving birth by cesarean as reported in the literature were assessed. Five hundred eighteen women were surveyed, of whom 58 had a cesarean birth. Statistical analyses revealed few significant differences between the two groups with regard to childbirth knowledge and attitudes, which may indicate that Latinas are different from the Anglo women discussed in the literature. In their postnatal assessment, 28 percent of the women giving birth by cesarean reported dissatisfaction with the experience, the majority regarded cesareans as "normal," and 11 percent thought they were at an advantage to have had cesarean births. These results suggest that cultural beliefs and attitudes may affect a woman's perceptions of the childbirth experience. The findings discussed here have implications for cross-cultural research on childbirth for childbirth educators and for health care providers working in multicultural settings.
\end{abstract}

\section{Introduction}

The national increase in the cesarean birth rate has been accompanied by growing popular and professional literature reporting a difference in women's perceptions of vaginal and cesarean births. Satisfaction with the childbirth experience is reported to be significantly lower among women delivering by cesarean $(1-10)$. These women tend to view their births as abnormal and having social stigma (6). They are also unlikely to have positive perceptions of the experience and consequently are more likely to report feelings of failure, anger, depression, fear,

Laura H. Cummins is a doctoral candidate in the Department of Anthropology, and Susan C.M. Scrimshaw is with the School of Public Health and Department of Anthropology at the University of California, Los Angeles. Patricia L. Engle is at the California Polytechnic State University at San Luis Obispo.

This study was funded by a grant from NICHD Division of Maternal and Child Health, HD RO1, HD 13796-02, to Susan C. M. Scrimshaw, principal investigator. resentment, guilt, self-blame, inadequacy, and disappointment (11).

It has also been suggested that cesarean birth interferes with initial mother-infant bonding. According to a study conducted by Marut and Mercer (6), mothers having cesarean delivery were less likely to have named their infants when interviewed 48 hours postpartum. In other studies women giving birth by cesarean have been reported as less likely than women giving birth vaginally to breastfeed their infants $(9,12)$.

Yet, in contrast to these findings, Bradley, Ross, and Warnyca (13) reported no significant differences between levels of anxiety, depression, or attitude toward the infant between women giving birth vaginally and those giving birth by cesarean, although the latter were more dissatisfied with the method of delivery.

The conflicting findings in the literature may be attributed to the instruments used and the types of questions asked $(13,14)$. The findings may also be complicated by the timing of interviews (15). Interviews conducted in a hospital setting may be too early and reflect a woman's desire to provide particular responses in that setting; interviews con- 
ducted at home at a later time may reflect more positive perceptions. Furthermore, women who give birth by cesarean are affected by extreme fatigue, pain, and discomfort (16). Thus, the reported differences between women based on type of delivery may merely reflect medical factors relating to the surgical birth and the subsequent postoperative period. An additional factor appears to have been overlooked, however; that is, the cultural context of birth. The findings reported in the literature may be complicated by a bias toward a predominantly Anglo, well-educated, middle-class sample who have particular expectations for childbirth and who are likely to express dissatisfaction when these expectations are not met. Culture is rarely explicitly mentioned in the majority of studies, particularly with regard to cesarean birth. Failure to consider this can be taken to mean that either culture is irrelevant, or that everyone believes and behaves in accordance with the dominant Anglo culture, which tends to be viewed as the norm. The anthropological literature has long drawn the connection between childbirth and culture (17-21). It is the cultural context that defines the norms that influence knowledge, attitudes, beliefs, expectations, and perceptions about the childbirth experience. It has been shown that lower socioeconomic groups are most likely to maintain traditional beliefs and behaviors (22).

This paper describes data from the UCLA Birth Project. Although the project focused on the cultural aspects of pregnancy and childbirth among low-income women of Mexican origin in Los Angeles and not specifically on cesarean birth, questions were asked that are appropriate to an analysis of cesarean birth among these women, and that suggest hypotheses for future research.

\section{Methods}

\section{Selection of Subjects}

From July of 1981 through September of 1982, 291 low-risk primiparous women of Mexican origin giving birth in two Los Angeles hospitals were interviewed once in the last six weeks of pregnancy and again during their postpartum hospital stay. This group is referred to as the longitudinal sample. All eligible patients from county clinics serving these hospitals and in the hospitals' prenatal clinics were interviewed. No private patients were included in order to control for lower socioeconomic status. Women who had a previous pregnancy that went beyond 20 weeks were excluded from the sample on the grounds that they would have had a previous birth experience similar enough to term delivery to bias their perceptions. To meet the lowrisk criteria, women with more than three prior abortions (either induced or spontaneous) or who had major complications of pregnancy (e.g., diabetes, hypertension, multiple fetuses, and eclampsia) were excluded. Those who developed complications after the initial interview at 34 or more weeks' gestation were retained in the sample.

Another 227 women who met the study criteria were interviewed only in the postpartum period, although relevant questions from the prepartum questionnaire were asked. This group is referred to as the postpartum sample (Table 1). All women who met the study criteria during the data-collection period were asked to participate. Although the postpartum sample was not located in the prenatal clinics, all but four women reported they had received some prenatal care elsewhere.

Data on the medical aspects of labor and delivery were abstracted from each woman's medical chart. There were few statistically significant differences between the longitudinal and postpartum samples, so the total sample of 518 women is discussed except when differences between the two are of importance (23).

\section{Procedures}

The prenatal interviews were conducted in hospital clinics by a team of trained Spanish-speaking interviewers. Interviewees were in the thirty-fourth week of pregnancy or beyond. The postnatal interviews were conducted 24 to 48 hours after delivery for the women who delivered vaginally and 48 to 96 hours after delivery for those who gave birth by cesarean.

\section{Measures}

First a series of open-ended questions was asked about childbirth in general; for example, "What comes to mind when you think about labor and delivery?" A second series of open-ended questions

Table 1. Sample

\begin{tabular}{lccc}
\hline & \multicolumn{2}{c}{ Longitudinal } & Postpartum \\
& Prepartum & Postpartum & Only \\
\hline Total identified & 406 & 372 & 237 \\
Interviewed & 372 & 291 & 227 \\
Declined & 34 & 1 & 10 \\
Not located & & & \\
$\quad$ postpartum & & 74 & \\
$\quad$ Not interviewed* & & 6 & \\
$\quad$ Total cases $\quad$ for analysis & & 291 & 227 \\
\hline
\end{tabular}

* Neonatal death (1), delivered at another hospital (5). 
was specifically about cesarean birth; for example, "Have you heard about cesarean sections?"; "What do you know about cesarean sections?" The women were also asked, "Of every 100 pregnant women, how many do you think have c sections?'"

The second part of the analysis was conducted postpartum and permitted comparison between the women giving birth by cesarean and those giving birth vaginally. The two groups were compared with regard to reports of their preparation for birth and prior experience with birth, including attendance at childbirth education classes. Specifically, the women were asked two questions: "Did you read or see anything about what birth will be like?" and "Did you attend any classes on childbirth?"

Two standardized scales, the maternal attitude toward pregnancy instrument (MAPI) (24) and the Spielberger state anxiety scale (25), were employed since they have been used previously with Latina populations. Two items on the MAPI ("Most women know how babies are born" and "Most women are unprepared for the birth of their first child') tested knowledge about childbirth. Responses on the Spielberger state anxiety scale after delivery were compared for the two groups of women. Comparisons were also made on responses to the women's postpartum semantic differential describing their childbirth experience and their assessment of their infants. Word pairs on the semantic differential for women included fast/slow, easy/difficult, bad/good, sick/healthy, pleasurable/ sad, passive/active, beautiful/ugly, calm/excitable, familiar/strange, humorous/serious, and responsible/irresponsible. Word pairs on the semantic differential for infants included small/large, beautiful/ ugly, sickly/healthy, calm/fussy, happy/sad, drowsy/alert, easy/difficult, boring/interesting, quiet/noisy, weak/strong, slow/fast, hot/cold, and soft/hard. The groups were also compared with respect to how much time they spent with their infant and how satisfied they were with this amount of contact.

Last, the women who gave birth by cesarean were asked a series of open-ended questions to elicit specific information on their perceptions of the cesarean birth experience; for example, "What did you think when they told you you needed to have a cesarean?"; "How would you describe your feelings about having had a cesarean?" A comparison to the women who gave birth vaginally could not be undertaken since these women were not asked to describe their childbirth experiences more specifically.

\section{Results}

Ninety-five percent of the total sample of women were born in Mexico. More than one-fourth had been in the United States less than one year, and 82 percent had been in the United States seven years or less. Eighty-five percent expressed a preference for receiving explanations in Spanish. Therefore, the sample represents newly arrived women who are still likely to be familiar with and influenced by the traditional beliefs and practices of Mexican culture while faced with American cultural pressures, practices, behaviors, and lifestyles.

Fifty-eight of the 518 women studied gave birth by cesarean (11\% of the total sample). This percentage compares with the 12 percent primary cesarean section rate reported for 1980 (26).

Table 2 lists some characteristics of the women experiencing a cesarean birth as compared to those giving birth vaginally. When these characteristics are compared, only age differs significantly between the groups. The mean age of women giving birth vaginally was 20.9 years compared with 22.6 years for those giving birth by cesarean $(P 0.016)$.

The women who participated in this study had very limited knowledge about childbirth, particularly with regard to cesarean birth. When interviewed prepartum there was no specific mention of cesarean birth by the longitudinal sample in response to the question, "What comes to mind when you think of labor and delivery?" When these women were asked the second open-ended ques-

Table 2. Characteristics of the Women Studied (longitudinal and postpartum samples combined)

\begin{tabular}{|c|c|c|}
\hline $\begin{array}{c}\text { Demographic } \\
\text { Variables }\end{array}$ & $\begin{array}{c}\text { Vaginal Birth } \\
(\%) \\
(\mathrm{n}=452)\end{array}$ & $\begin{array}{c}\text { Cesarean Birth } \\
(\%) \\
(\mathrm{n}=58)\end{array}$ \\
\hline Born in Mexico & 94 & 97 \\
\hline \multicolumn{3}{|c|}{ Years in the United States } \\
\hline Less than 1 & 26 & 25 \\
\hline Less than 7 & 82 & 82 \\
\hline \multicolumn{3}{|l|}{ Marital status } \\
\hline Married & 64 & 60 \\
\hline Single & 35 & 39 \\
\hline Mean age (yrs) & 20.9 & $22.6^{*}$ \\
\hline Baby was planned & 58 & 60 \\
\hline \multicolumn{3}{|l|}{ Desire for more } \\
\hline than 1 child & 84 & 84 \\
\hline \multicolumn{3}{|c|}{ Attended childbirth education classes } \\
\hline No & 87 & 78 \\
\hline Yes & 13 & $22 \dagger$ \\
\hline
\end{tabular}


tion about concerns during labor and delivery, only 7 percent mentioned cesarean birth.

In response to the question asking if they had heard about cesarean sections, 77 percent of the 452 women responding answered affirmatively; only 21 percent claimed not to have heard about cesareans. Table 3 lists the responses of 279 women to the question, "What do you know about cesarean sections?" asked before delivery of the longitudinal sample only. The open-ended responses were coded into six categories: 1) the baby's not coming out right, or incorrect position of the baby; 2) a cesarean is an operation done to take out the baby; 3 ) cesarean is a dangerous procedure, performed when the birth is difficult; 4) failure to make progress in birthing; 5) pain or ugliness of the procedure; and 6) cesarean is only done when necessary for the mother or the baby. The most frequent response referred to the baby's not coming out right/not in the correct position/can't have a normal delivery (41\%). Knowledge of cesarean section as an operation was reported by only 25 percent.

Two hundred sixty women in the longitudinal sample responded to the question, "Of every 100 pregnant women, how many do you think have csections?" The modal response was 50 cesarean births per 100; the mean response was 25 per 100 .

The second part of the analysis revealed no difference between the women who gave birth by cesarean and those who gave birth vaginally in response to the question, "Did you read or see anything about birth." In each group, a little more than half of the respondents (55\%) indicated that they had read or seen something about birth. Yet

Table 3. What Do You Know About Cesarean Sections? (asked of longitudinal sample only)

\begin{tabular}{|c|c|c|}
\hline Response & $\begin{array}{l}\text { Number } \\
(\mathrm{n}=279)\end{array}$ & $\%$ \\
\hline $\begin{array}{l}\text { They aren't coming out right } \\
\text { Baby's not in the correct position }\end{array}$ & & \\
\hline $\begin{array}{l}\text { Very big baby } \\
\text { Transverse/breech/the head is not up } \\
\text { Baby is not breathing } \\
\text { Can't have a normal delivery }\end{array}$ & 115 & 41 \\
\hline $\begin{array}{l}\text { Can't have a normal delivery } \\
\text { Operation done to take out the baby }\end{array}$ & 69 & 25 \\
\hline $\begin{array}{l}\text { It's dangerous } \\
\text { When it's a difficult birth } \\
\text { When the woman is narrow }\end{array}$ & 37 & 13 \\
\hline $\begin{array}{l}\text { No progress } \\
\text { Nothing is happening } \\
\text { The baby isn't coming out }\end{array}$ & 26 & 9 \\
\hline Painful/ugly & 19 & 7 \\
\hline $\begin{array}{l}\text { Done only when necessary for the } \\
\text { baby or the mother }\end{array}$ & 13 & 5 \\
\hline
\end{tabular}

with regard to attendance at childbirth education classes, 22 percent of the women who gave birth by cesarean had attended such classes compared to 13 percent of those who delivered vaginally. While not statistically significant $\left(\chi^{2}=3.35 ; d f=1 ; P 0.067\right)$, the difference approaches significance and could be regarded as a trend to explore in future research. Indeed, in response to two items on the MAPI, women giving birth by cesarean clearly showed they were more knowledgeable about childbirth. These mothers were more likely to agree with the statement, "Most women know how babies are born" ( $\left.\chi^{2}=11.177 ; d f=3 ; P 0.01\right)$ and more likely to disagree with the statement, "Most women are unprepared for the birth of their first child" $\left(\chi^{2}=\right.$ $7.375 ; d f=3 ; P 0.06$ ), which may indicate increased knowledge and preparation as acquired in childbirth education classes. Attendance at these classes was significantly related to the number of years a woman had lived in the United States. The longer a woman had lived in this country, the more likely she was to have attended such classes $(t=$ -2.28, $P$ 0.025).

Although not highly significant, the overall postpartum anxiety score was higher for women who gave birth by cesarean $(t=-1.87, P 0.062)$. When interviewed postpartum, the two groups showed a significant difference in their responses to only two items on the Spielberger state anxiety scale. Women giving birth by cesarean reported themselves to be less calm $\left(\chi^{2}=9.665 ; d f=3\right.$; $P$ 0.0216) and less rested $\left(\chi^{2}=13.00 ; d f=3\right.$; $P$ 0.0046). They were also more concerned about possible misfortune $\left(\chi^{2}=6.968 ; d f=3 ; P 0.07\right)$. While this is not a significant difference, it is a possible trend in the expected direction given that these women had just experienced a surgical birth.

Minor differences were reported in response to two pairs of words in the semantic differential for the infant scale items: "beautiful/ugly" $\left(\chi^{2}=\right.$ $12.90 ; d f=6 ; P 0.04)$ and "weak/strong" $\left(\chi^{2}=\right.$ $11.26 ; d f=5 ; P 0.08$ ), with cesarean mothers reporting their infants as uglier and weaker. There was no relationship between women's attitude toward their infants and difficulty of labor and delivery, as indicated by the number of complications and the likelihood of having a cesarean (27).

Self-reports revealed that women giving birth by cesarean spent significantly less time $(t=7.85$, $P 0.01)$ with their infants than did those who gave birth vaginally (mean 4.7 vs 7.5 hours; mode 0.0 vs 9.0 hours). More than half of the cesarean mothers were satisfied with the amount of time they had with their infants, although 31 percent expressed 
some dissatisfaction, saying they would have liked more time. In comparison, 74 percent of the women delivering vaginally were satisfied with the amount of time they had with their baby, and only 17 percent expressed a desire for more time. This was a significant difference in satisfaction with the amount of time the women spent with their infants $\left(\chi^{2}=9.113 ; d f=2 ; P 0.01\right)$.

Although 82 percent of the women interviewed prenatally planned to breastfeed, those giving birth by cesarean were less likely to have initiated breastfeeding at the time of interview $\left(\chi^{2}=21.71\right.$; $P$ 0.001) (28).

The 58 women giving birth by cesarean were asked to describe their experience in a series of open-ended questions 48 to 96 hours postpartum. To the question, "What did you think when they told you that you needed to have a cesarean?", the two most frequent responses were "afraid for the baby" (39\%) and "relief/glad it's over" (31\%). Only 12 percent were angry or blamed the doctor or hospital.

In describing their feelings about having had a cesarean birth in comparison to their expectation for a vaginal birth, 53 percent reported that they felt the cesarean birth was normal - they just could not have a vaginal birth. Twenty-eight percent expressed disappointment. Eleven percent regarded themselves as lucky; they thought vaginal births were harder. Only eight percent described themselves as feeling bad, having failed, or guilty (Table 4).

\section{Discussion}

Although the literature indicates a significant difference between women who give birth vaginally and those who give birth by cesarean in their assessment of the childbirth experience, we question the applicability of these findings to all ethnic groups based on our study of a group of lowincome women of Mexican origin in Los Angeles. Although the power of this study was small, it suggests that cultural beliefs and attitudes affect perceptions about the childbirth experience, particularly cesarean birth, and thus should be considered in future research.

The participants in this study had very limited knowledge about childbirth (28). This was especially true with respect to cesarean birth. Although the women claimed to have heard about cesareans, it was not mentioned as a concern.

Attendance at childbirth education classes and knowledge about childbirth events appears to be the most interesting difference between the two groups. The women who gave birth by cesarean
Table 4. Feelings About Having Had a Cesarean Birth (asked only of women in the postpartum sample who experienced a cesarean birth)

\begin{tabular}{lrr}
\hline \multicolumn{1}{c}{ Response } & $\begin{array}{c}\text { Number } \\
(\mathrm{n}=57)\end{array}$ & $\%$ \\
\hline Normal, just couldn't have a vaginal & & \\
$\quad$ birth & 30 & 53 \\
Disappointed/lost the birth experience & 16 & 28 \\
Lucky, a vaginal birth is harder & 6 & 11 \\
Bad/failed/guilty & 5 & 8 \\
\hline
\end{tabular}

were more likely to have attended childbirth education classes, although the sample as a whole did not indicate much participation in such classes. While these classes are offered throughout Los Angeles, they are geared to a Caucasian, educated, middleand upper-class population, and are rarely attended by Latina women. Nurses working in the postpartum area of one of the hospitals where this study was conducted were interviewed in August 1985 as to their perceptions of how different ethnic groups assess vaginal and cesarean births (29). Some of the nurses reported, "Mexican women don't attend these [childbirth education] classes . . . they get what they need to know from other women" (29). Transmission of childbirth knowledge from woman to woman is documented in the anthropological literature $(20,30)$; however, there are undoubtedly other reasons why childbirth education classes are not popular among Latinas. Our pilot work revealed their inability to attend these classes because of employment, the location and time of classes, the availability of their partner to accompany them, as well as fear of childbirth (28). Language did not appear to be a factor since even when the classes were offered in Spanish, few women attended. It may also be that among Mexican women, discussing childbirth with strangers is considered improper until after a woman has had her first child.

It is not clear why the more knowledgeable women had cesarean births, although we speculate that communication with health care providers may have played a role. The women who attended childbirth education classes had lived in the United States longer, probably spoke more English, and may have complained or even asked for a cesarean if labor was perceived to be prolonged. During our labor and delivery observations of over 100 women in the pilot study for the UCLA Birth Project, we noted that women who spoke less English were less assertive, communicated less, had less interaction with the hospital staff, and appeared to receive fewer interventions. The hospital staff may uncon- 
sciously have postponed a decision to perform a cesarean, allowing more time for a vaginal birth to occur. This is an area for further research to determine if it is an artifact of this study or if it is borne out in other samples.

The women giving birth by cesarean regarded a cesarean birth as "normal" (53\%) or even regarded themselves as "luckier" than women who give birth vaginally (11\%) since they perceived a vaginal birth as being harder. In fact, the women in this study expressed a high degree of concern about pain at delivery with high levels of pain at delivery expected (31). These positive perceptions of cesarean birth clearly contrast with the assessment of Anglo women reported in the literature. This may be due in part to Mexican women's fear of childbirth; a cesarean birth may be perceived as helping a woman to escape the pain expected in a vaginal birth. Our labor and delivery observations in the pilot study for the UCLA Birth Project lend further support to this positive view of cesarean birth. Many women expressed the feeling that when labor was too difficult, a cesarean was an alternative, and some actually requested a cesarean. Those who were discouraged with their labor and the pain also tended to expend less effort in the second stage of labor.

Furthermore, because cesarean birth is reported to be more common among upper classes (32), these lower-class Latinas may actually view having a cesarean as a "status symbol." Although the high modal response of 50 cesarean births per 100 pregnant women to the question on the frequency of cesarean births may reflect some confusion over the question, it may also be related to lack of knowledge about cesarean births, or the perception that cesarean births are normal and are to be expected in high numbers.

Any significant differences between the two groups with regard to the items on the Spielberger state anxiety scale and the semantic differential measurements may be a result of postoperative factors rather than an actual difference in the assessment of the childbirth experience. Pain, discomfort, reactions to medication, and the inability to function normally after surgery may have affected responses to the questions that were asked. It is interesting to note that the women giving birth by cesarean reported themselves to be less calm and more concerned about possible misfortune. This may reflect a concern over economic issues, since cesareans are more costly, particularly if they were self-pay patients. Recovery after the cesarean birth might also be a concern if the woman had plans to return to work. However, the fact that the interviews were conducted at a later time than most of those reported in the literature might account for the lack of significant differences between the two groups.

It was evident from the data that the women giving birth by cesarean spent significantly less time with their infants. This type of delivery delays the initiation of mothering behavior, which may have long-term effects $(33,34)$. Although the women who gave birth by cesarean were less likely to have begun breastfeeding by the time of the interview, this is more likely a reflection of hospital policies and practices that often separate cesarean mothers from their infants, as well as the contraindication of nursing due to maternal fever or the effects of drugs taken after surgery, rather than a conscious failure on the part of the new mother to initiate mothering behavior. Scrimshaw, Engle, Arnold, and Haynes (23) reported that the women in this study who spent more time with their babies were also most likely to breastfeed. As there was no relationship between prepartum breastfeeding plans and type of delivery, the amount of time spent with the infant emerges as an important consideration in breastfeeding. The anthropological literature supports this finding, since breastfeeding is encouraged in the traditional Mexican and Mexican-American family $(20,30)$.

In conclusion, cesarean birth among women of Mexican origin in Los Angeles did not appear to be an unsatisfying, psychologically negative experience. Although some mothers described their feelings in negative terms (specifically, fear) over half perceived a cesarean as a normal way of birthing; 11 percent felt lucky to have had a cesarean, while 8 percent reported feeling bad, guilty, or that they had failed.

The cultural context of cesarean birth must be understood. In the United States where diverse ethnic groups use the same medical system, it should not be assumed that all groups of women have the same knowledge and expectations regarding childbirth or view cesarean birth negatively. Our study suggests that cesarean birth may not be negatively perceived by all groups of women. A cesarean may not necessarily be considered a threat, and the psychological response to this type of delivery may therefore be more positive. Consequently, satisfaction with the birth experience and initiation of mothering behavior may not be diminished by women experiencing a cesarean.

\section{References}

1. Arms S. Immaculate Deception. Boston: Houghton Mifflin, 1977.

2. Cohen NW. Minimizing the emotional sequelae of cesarean childbirth. Birth Fam J 1977;4:114-119. 
3. Donovan B. The Cesarean Birth Experience. Boston: Beacon Press, 1977.

4. Affonso D, Stichler J. Exploratory study of women's reactions to having a cesarean birth. Birth Fam J 1978;5:88-94.

5. Affonso D, Stichler J. Impact on women: Feelings and perceptions. In: Affonso D, ed. Impact of Cesarean Childbirth. Philadelphia: FA Davis, 1981:39-61.

6. Marut JS, Mercer RT. Comparison of primiparas' perceptions of vaginal and cesarean births. Nurs Res 1979;28: 260-266.

7. Lipson JG, Tilden VP. Psychological integration of the cesarean birth experience. Am J Orthopsychiatry 1980;50: 598-609.

8. Tilden VP, Lipson JG. Cesarean childbirth: Variables affecting psychological impact. West J Nurs Res 1981;3:127148.

9. Cranley MS, Hedahl KJ, Pegg SH. Women's perceptions of vaginal and cesarean deliveries. Nurs Res 1983;32:1015.

10. Cohen NW, Estner LJ. Silent Knife. South Hadley, MA: Bergin and Garvey, 1983.

11. Birdsong LS. Loss and grieving in cesarean mothers. In: Fitzgerald KC, ed. The Cesarean Experience. New York: Appleton-Century-Crofts, 1981:187-209.

12. Entwisle D, Doering S. The First Birth. A Family Turning Point. Baltimore: Johns Hopkins University Press, 1981.

13. Bradley C, Ross SE, Warnyca J. A prospective study of women's attitudes and feelings following cesarean and vaginal births. Birth 1983;10:79-83.

14. Shearer EC. How do parents really feel after cesarean birth. Birth 1983;10:91-92.

15. Erb L, Hill G, Houston D. A survey of parents attitudes toward their cesarean births in Manitoba hospitals. Birth 1983;10:85-91.

16. Marieskind M. An Evaluation of Cesarean Section in the United States. Washington, DC: U.S. Department of Health, Education and Welfare, 1979.

17. Ford CS. A Comparative Study of Human Reproduction. New Haven, CT: Human Relations Area Files Press, 1945.

18. Mead M, Newton N. Cultural patterning of perinatal behavior. In: Richardson SA, Guttmacher AF, eds. Childbearing: Its Social and Psychological Aspects, New York: Williams \& Wilkins, 1964.

19. Newton N. Pregnancy, childbirth and outcome: A review of patterns of culture and future needs. In: Richardson SA,
Guttmacher AF, eds. Childbearing: Its Social and Psychological Aspects. New York: Williams \& Wilkins, 1964.

20. Jordan B. Birth in Four Cultures. Montreal: Eden Press, 1980.

21. Kay MA. Anthropology of Human Birth. Philadelphia: FA Davis, 1982.

22. Moustafa A, Weiss G. Health Status and Practices of Mexican-Americans. Mexican-Study Project, Advance report no. 11, UCLA, February 1968.

23. Scrimshaw SCM, Engle PL, Arnold A, Haynes K. Factors affecting breastfeeding among Mexican women in Los Angeles. Am J Public Health 1987;77(4):467-470.

24. Blau A, Cohen J, Weldowitz J. MAPI: A research instrument for psychogenic obstetrical complications. Arch Gen Psychiatry 1964;10:325-329.

25. Spielberger CD, Gorsuch RL, Lushene RE. STAI Manual for the State Trait Anxiety Inventory. Palo Alto, CA: Consulting Psychologists, 1970.

26. Taffel SM, Placek PJ, Less T. Trends in the U.S. cesarean section rate and reasons for the 1980-1985 rise. Am J Public Health 1987;77(8):955-959.

27. Engle PL, Scrimshaw SCM, Smidt R. Sex differences in attitude towards newborn infants among women of Mexican origin. Med Anthropol 1984;8:133-144.

28. Scrimshaw SCM, Engle PL, Horsley K. Use of prenatal care services by women of Mexican origin and descent in Los Angeles. Unpublished manuscript, 1985.

29. Cummins LH. The cesarean birth experience among Mexican women in Los Angeles: An exploratory discussion. Unpublished master's thesis, UCLA, 1985.

30. Kay MA. The Mexican American. In: Clark A, ed. Culture/Childbearing/Health Professionals. Philadelphia: FA Davis, 1982.

31. Engle PL, Scrimshaw SCM, Zambrana RE, DunkelSchetter C. Prenatal and postnatal anxiety in Mexican women giving birth in Los Angeles. Health Psychol (in press).

32. Hurst M, Summey PS. Childbirth and social class: The case of cesarean delivery. Soc Sci Med 1983;18:621-631.

33. Klaus $\mathrm{MH}$, Jerauld R, Kreger NC, et al. Maternal attachment: Importance of the first postpartum days. $N$ Engl $J$ Med 1972;286:460-463.

34. Kennell JH, Jerauld R, Wolfe H, et al. Maternal behavior one year after early and extended contact. Dev Med Child Neurol 1974;16:172-179. 